\title{
The Clinical Role of SRSF1 Expression in Cancer: A Review of the Current Literature
}

\author{
Arturo Lo Giudice $1,2, *\left(\mathbb{0}\right.$, Maria Giovanna Asmundo ${ }^{1, *}$, Giuseppe Broggi ${ }^{3}{ }^{\circledR}$, Sebastiano Cimino ${ }^{1}$, \\ Giuseppe Morgia ${ }^{1}$, Ettore Di Trapani ${ }^{2}$, Stefano Luzzago ${ }^{2,4}$, Gennaro Musi ${ }^{2,4}$, Matteo Ferro ${ }^{2}(\mathbb{D}$, \\ Ottavio de Cobelli ${ }^{2,4}$ and Giorgio I. Russo ${ }^{1, *(\mathbb{D}}$
}

1 Urology Section, Department of Surgery, University of Catania, 95123 Catania, Italy; ciminonello@hotmail.com (S.C.); giuseppe.morgia@unict.it (G.M.)

2 Department of Urology, European Institute of Oncology, IRCCS, 20141 Milan, Italy; ettore.ditrapani@ieo.it (E.D.T.); stefano.luzzago@ieo.it (S.L.); gennaro.musi@ieo.it (G.M.); matteo.ferro@ieo.it (M.F.); ottavio.decobelli@ieo.it (O.d.C.)

3 Department of Medical and Surgical Sciences and Advanced Technologies "G. F. Ingrassia", Anatomic Pathology, University of Catania, 95123 Catania, Italy; giuseppe.broggi@gmail.com

4 Department of Oncology and Haemato-Oncology, University of Milan, 20122 Milan, Italy

* Correspondence: arturologiudice@gmail.com (A.L.G.); mariagiovannaasmundo@gmil.com (M.G.A.); giorgioivan1987@gmail.com (G.I.R.)

Citation: Lo Giudice, A.; Asmundo, M.G.; Broggi, G.; Cimino, S.; Morgia, G.; Di Trapani, E.; Luzzago, S.; Musi, G.; Ferro, M.; de Cobelli, O.; et al. The Clinical Role of SRSF1 Expression in Cancer: A Review of the Current Literature. Appl. Sci. 2022, 12, 2268. https://doi.org/10.3390/app12052268

Academic Editor: Francesca Silvagno

Received: 19 December 2021

Accepted: 18 February 2022

Published: 22 February 2022

Publisher's Note: MDPI stays neutral with regard to jurisdictional claims in published maps and institutional affiliations.

Copyright: (c) 2022 by the authors. Licensee MDPI, Basel, Switzerland. This article is an open access article distributed under the terms and conditions of the Creative Commons Attribution (CC BY) license (https:// creativecommons.org/licenses/by/ $4.0 /)$.

\begin{abstract}
Background: SFRS1 is a member of the splicing factor protein family. Through a specific sequence of alteration, SRSF1 can move from the cytoplasm to the nucleus where it can work autonomously as a splicing activator, or as a silencer when interacting with other regulators. Alternative splicing (AS) is a fundamental biological process that ensures protein diversity. In fact, different proteins, produced by alternative splicing, can gain different and even antagonistic biological functions. Methods: Our review is based on English articles published in the MEDLINE/PubMed medical library between 2000 and 2021. We retrieved articles that were specifically related to SRSF1 and cancers, and we excluded other reviews and meta-analyses. We included in vitro studies, animal studies and clinical studies, evaluated using the Medical Education Research Study Quality Instrument (MERSQI) and the Newcastle-Ottawa Scale-Education (NOSE). Result: SRSF1 is related to various genes and plays a role in cell cycle, ubiquitin-mediated proteolysis, nucleotide excision repair, p53 pathway, apoptosis, DNA replication and RNA degradation. In most cases, SRSF1 carries out its cancer-related function via abnormal alternative splicing (AS). However, according to the most recent literature, SRSF1 may also be involved in mRNA translation and cancer chemoresistance or radio-sensitivity. Conclusion: Our results showed that SRSF1 plays a key clinical role in tumorigenesis and tumor progression in several types of cancer (such as Prostate, Lung, Breast, Colon, Glioblastoma), through various mechanisms of action and different cellular pathways. This review could be a starting point for several studies regarding the biology of and therapies for cancer.
\end{abstract}

Keywords: SRSF1; prostate; glioblastoma; lung; colon; breast; cancer; splicing

\section{Introduction}

SRSF1 (Serine and Arginine Rich Splicing Factor 1), also known as ASF, SF2, SF2P33 or SFRS1, is a member of the splicing factor protein family. This family is composed of twelve members, each containing a characteristic carboxyl-terminal domain rich in arginine and SR (serine residues) domains and one or two RRM (RNA-recognition motifs) [1]. Moreover, the SR (Serine Residues) protein is involved in gene expression pathways affecting both pre-mRNA as well as mRNA mediating mRNA decay, mRNA export and translation, miRNA processing, protein stimulation and the nucleolar stress response [2,3]. In fact, Anczuków, O. et al. observed that SRSF1 can work autonomously as a splicing activator, while it requires interaction with other splicing regulators to function as a silencer [3]. According to the study conducted by Ngo et al., SRSF1 is initially phosphorylated by SRPK1 
(SR protein-specific-kinase 1) on a precise serine residue contained in the SR domain [4]; due to its hypophosphorylation and through its association with a specific transportin, the protein is imported into nuclear storage. Therefore, SRSF1 recruitment to active sites of transcription, where it is involved in splicing regulation, requires a second phosphorylation, which is carried out by CLK (CDC-like kinase 1) [5,6]. Therefore, SRSF1, bound to spliced-mRNA, is exported into the cytoplasm through its interaction with the TAP/NXF1 receptor [7]; this evidence is shown in Figure 1.

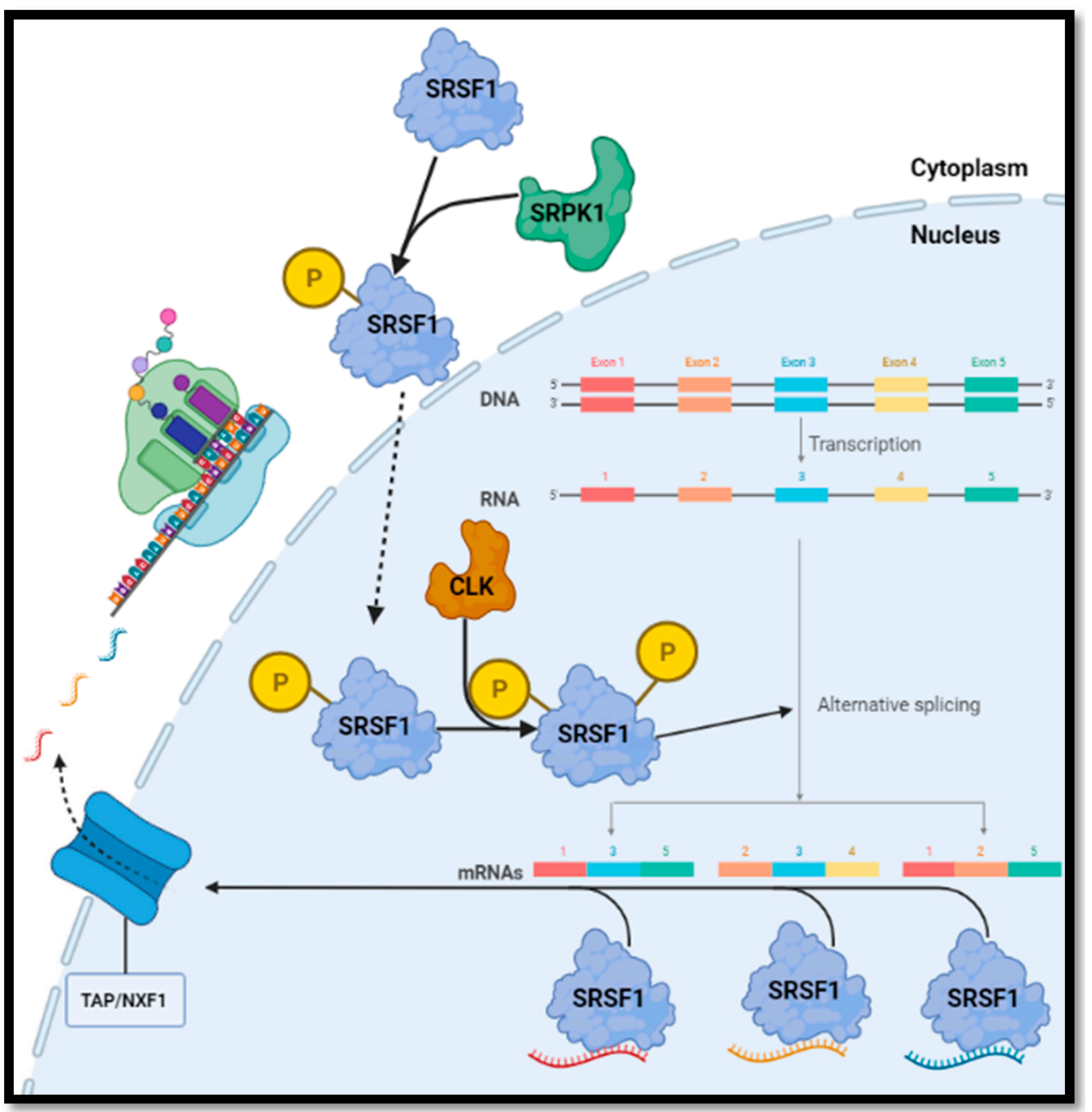

Figure 1. Alternative splicing event mediated by SRSF1. Created with Biorender.

Alternative splicing is a fundamental biological process that ensures protein diversity. In fact, different proteins, produced by alternative splicing, can gain different, and even antagonistic, biological functions. As a confirmation of this, through alternative splicing, about $95 \%$ of the human genome produces multiple mRNA transcripts by the differential inclusion or exclusion of exons [6]. There is much evidence of an association between alternative splicing and cancer insurgence. Interesting, many genes involved in proliferation, invasiveness, and programmed cell death are modulated by alternative splicing and, as a consequence of failure to accurately recognize the splicing site, may generate mRNA variants encoding for deleterious protein-isoforms that contribute to tumor phenotypes [8,9]. Some of these mutations are caused by changes that occurred at the splicing site of tumor-suppressor genes, while others are caused by alterations in splicing factor proteins [10]. Furthermore, according to the study conducted by Das et al., SRSF1, primarily driven by the hyperactivation of the transcription factor MYC, is overexpressed in a wide range of cancers and plays oncogenic roles through 
its control of AS of cancer related genes [11]. Moreover, changes in the expression of SRSF1 are potentially involved in splicing misregulation in several diseases including Retinitis Pigmentosa 4 and Homocystinuria [12].

In recent years, scientific interest has been focused on SRSF1 as an alternative splicing regulator and as a crucial molecular biomarker and possible therapeutic target.

The aim of this narrative review is to give an update about the clinical role of SRSF1 on different cancers and to clarify the state of the literature, to stimulate further studies about alternative spicing in cancer and this important splicing factor protein.

\section{Materials and Methods}

\subsection{Data Source}

We performed the literature review on MEDLINE/PubMed medical library. We used the following keywords: "Cancer and SRSF1", "Prostate cancer and SRSF1", "Breast cancer and SRSF1", "Lung cancer and SRSF1", "Colorectal cancer and SRSF1", "Colon cancer and SRSF1", "Rectal cancer and SRSF1", "Glioma and SRSF1", "Esophagus cancer and SRSF1", "Hepatocarcinoma and SRSF1", "Leukemia and SRSF1", "Ovarian cancer and SRSF1", "Pancreatic cancer and SRSF1", "Kidney cancer and SRSF1", "Stomach cancer and SRSF1", "Head and Neck cancer and SRSF1". Finally, we employed a snowball method to search for articles not identified in the first search.

\subsection{Inclusion Criteria}

We limited the search to articles published in English between 2000 and 2021. We retrieved articles that specifically related to SRSF1 and cancers; we excluded other reviews and meta-analyses. Articles were progressively screened at the title, abstract and fulltext level.

\subsection{Screening}

Two researchers (A.L.G. and M.G.A.) selected and reviewed the abstracts responding to precise qualitative criteria and according to their background and conclusions; those that met the inclusion criteria were printed and read in full. Screening of the articles began with the examination of the methodology, then bioinformatic studies, in vitro studies, in vivo animal studies and clinical studies were included and evaluated using the MERSQI (Medical Education Research Study Quality Instrument) and the NOS-E (Newcastle-Ottawa Scale Education) $[13,14]$.

\section{Results}

\subsection{Preliminary Results}

At the end of the screening, 23 articles were selected for the review and categorized for author, year, topic, type of study and for MERSQI and NOS-E, as specified in Section 2.3.

Most of studies selected for the review were about lung cancer (eight studies), followed by breast cancer, prostate cancer and glioblastoma multiforme.

All the studies were classified by topic and type of work as bioinformatic study, in vitro study, in vivo animal study or clinical study. We divided the type of studies by topic.

Most of the studies included were clinical (11 studies) or in vitro studies (11 studies; also, some studies were conducted by multiple approaches, not only as clinical, in vivo, in vitro or bioinformatic studies.

We evaluated all clinical studies through MERSQI and NOS-E scores, as specified in methods; all the scores were high.

Table 1 reports the list of articles included in our review with all the characteristics previously summarized. 
Table 1. Summary table. All the characteristics of the studies included, where reported.

\begin{tabular}{|c|c|c|c|c|c|c|c|c|c|}
\hline Num & Authors & Year & Topic & Bioinformatic & In Vitro & In Vivo Animal & Clinical & MERSQI & NOS-E \\
\hline [1] & Bates, Do. et al. & 2016 & Prostate cancer & & $X$ & & & NA & NA \\
\hline [2] & Gao, X. et al. & 2019 & Prostate cancer & & $x$ & $x$ & & NA & NA \\
\hline [3] & Guan, X. et al. & 2020 & Prostate cancer & & & & $X$ & 16 & 10 \\
\hline [4] & $\begin{array}{l}\text { Broggi, G. et al. } \\
\text { SUM }\end{array}$ & 2021 & $\begin{array}{c}\text { Prostate cancer } \\
4\end{array}$ & & & & $X$ & 16 & 10 \\
\hline [5] & Anczuków, O. et al. & 2015 & Breast cancer & & $x$ & & & NA & NA \\
\hline [6] & Vaklavas, C. et al. & 2019 & Breast cancer & & $X$ & & & NA & NA \\
\hline [7] & Du, J.X. et al. & 2021 & Breast cancer & & & & $X$ & 16 & 10 \\
\hline [8] & $\begin{array}{l}\text { Oh, J. et al. } \\
\text { SUM }\end{array}$ & 2021 & $\begin{array}{c}\text { Breast cancer } \\
4\end{array}$ & & & & $x$ & 16 & 10 \\
\hline [9] & Shultz, J.C. et al. & 2011 & Lung cancer & & $x$ & & & NA & NA \\
\hline [10] & Gout, S. et al. & 2012 & Lung cancer & & & & $x$ & 16 & 10 \\
\hline [11] & Jiang, L. et al. & 2016 & Lung cancer & & & & $x$ & 16 & 10 \\
\hline [12] & Sheng, J. et al. & 2018 & Lung cancer & & $x$ & $X$ & & NA & NA \\
\hline [13] & Martínez-Terroba, E. et al. & 2018 & Lung cancer & & & & $x$ & 16 & 10 \\
\hline [14] & Chang, H.L. et al. & 2019 & Lung cancer & & $x$ & & & NA & NA \\
\hline [15] & Wang, J. et al. & 2020 & Lung cancer & & $x$ & & & NA & NA \\
\hline [16] & $\begin{array}{l}\text { Lv, Y. et al. } \\
\text { SUM }\end{array}$ & 2021 & $\begin{array}{c}\text { Lung cancer } \\
\mathbf{8}\end{array}$ & & $x$ & & & NA & NA \\
\hline [17] & Sheng, J. et al. & 2017 & Colorectal cancer & $x$ & & & & NA & NA \\
\hline [18] & Chen, L. et al. & 2017 & Colorectal cancer & & $x$ & $x$ & $x$ & 16 & 10 \\
\hline [19] & $\begin{array}{l}\text { Liu, H. et al. } \\
\text { SUM }\end{array}$ & 2021 & $\begin{array}{c}\text { Colorectal cancer } \\
3\end{array}$ & & & & $x$ & 16 & 10 \\
\hline [20] & Barbagallo, D. et al. & 2018 & $\begin{array}{l}\text { Glioblastoma } \\
\text { multiforme }\end{array}$ & & & & $X$ & 16 & 10 \\
\hline [21] & Barbagallo, D. et al. & 2019 & $\begin{array}{l}\text { Glioblastoma } \\
\text { multiforme }\end{array}$ & & & & $X$ & 16 & 10 \\
\hline \multirow[t]{2}{*}{ [22] } & $\begin{array}{c}\text { Barbagallo, D. et al. } \\
\text { SUM }\end{array}$ & 2021 & $\begin{array}{l}\text { Glioblastoma } \\
\text { multiforme } \\
3\end{array}$ & & $x$ & & & NA & NA \\
\hline & & & & $\underset{1}{\text { SUM }}$ & $\begin{array}{c}\text { SUM } \\
11\end{array}$ & $\underset{3}{\text { SUM }}$ & $\begin{array}{c}\text { SUM } \\
11\end{array}$ & & \\
\hline
\end{tabular}

\subsection{Review}

\subsubsection{Prostate}

The increasing interest in SRSF1 led to studies about the most frequent cancers, obviously prostate cancer as the first cancer in men has been investigated in recent years. There is not a large number of studies yet, however, some literature findings are interesting and indicate that SRSF1 could also play a role in this tumor.

Some studies have demonstrated the higher expression of VEGF in PCa cells $[15,16]$, furthermore, a study published in 2016 found a higher expression of SRSF1 and SRPK1 (Serine Arginine Protein Kinase-1) in malignant Pca cells, as well as demonstrating that SRPK1 plays a role in the alternative splicing of VEGF to a more angiogenetic isoform (VEGF165b) leading to faster cancer growth [17]. As is well known, VEGF expression is regulated by the alternative splicing factor SRSF1, which is, in turn, regulated by SRPK phosphorylation $[18,19]$.

In 2020 Gao et al. published a paper that investigated the role of HSD17B2 (7ßHydroxysteroid dehydrogenase 2) in PCa. In their in vitro study, performed on culture and in vivo PCa cells using a xenograft model in mice, they found that HSD17B2 expression was inversely proportional to PCa development and progression; in fact, it was expressed more in benign tissue than in adjacent tumor tissue. The authors suspected that higher levels of HSD17B2 could suppress the synthesis of DHT (dihydrotestosterone) from androstenedione, which is well known as a PCa stimulating hormone, thus they knocked down HSD17B2 from a cell line and proved that the cell line without HSD17B2 had higher DHT synthesis. Furthermore, some authors found a higher expression of SRSF1 in PCa cells that expressed lower levels of HSD17B2 and, moreover, found a correlated presence of two isoforms of HSD17B2, the middle length (M) isoform and the short length (S) isoform. These isoforms were unable to interact with androgens and the authors demonstrated their capacity of degradation of HSD17B2 wild type (L, long, isoform). In conclusion, these authors affirmed that SRSF1 could modulate an alternative splicing event on PCa cells leading to 
the synthesis of M and S HSD17B2 isoforms that do not suppress DHT synthesis, promoting cancer progression and development in vitro and in the xenograft model in vivo [20].

In 2020, Guan et al. performed a WGS (Whole Genome Sequencing) and RNA sequencing on tissues collected from patients affected by mCRCP (Castration-Resistant Prostate Cancer), to investigate if any genomic changes occurred. Based on their response to chemotherapy, patients were divided into two groups: Enzalutamide-resistant and Enzalutamide-naive. The authors found that there was no statistical difference in copy gain events between the genomes of the two groups of patients; therefore, the most important differences were revelaed in the copy loss of genes. In particular, it was intriguing to find that the copy loss occurs in 17q22; this cytoband, in fact, is where SRSF1 and RNF43 genes are located. It is even more interesting to detect that the loss of these two genes was found in enzalutamide-resistant patients only. The authors then performed a survival analysis stratified by the deletion of SRSF1 and RNF43 and revealed that the deletion of 17q22 was related to poor prognosis and OS (overall survival). After they performed an MR (Master Regulator) analysis, an algorithm to predict variation in regulations related to a specific deletion, they identified that the loss of 17q22 containing SRSF1 and RNF43 could positively influence the activity of PLK1, AKT1 and cyclin-dependent kinases (CDK1 and CDK2) playing a well-known role in cell-cycle and tumorigenesis in prostate cancer [21]. In conclusion, the authors found a likely clinical role of SRSF1 and RNF43 in prostate cancer; resistance to enzalutamide therapy and reduction of OS.

Our group published a paper in 2021 reporting the construction of TMA (tissue microarray) blocks with a semi-automatic tissue microarrayer, the Galileo TMA CK3500 (Integrated System Engineering, Milan, Italy). We analyzed the clinical data of 368 patients who had undergone surgery for PCa or BPH (Benign Prostate Hyperplasia) in a single institution and obtained samples of tissues from paraffin blocks to construct the TMA blocks; we than performed an IHC (immunohistochemistry) analysis, where pathologists did not know clinical data of patients. We evaluated the expression of multiple proteins and SRSF1, we also evaluated MVD (microvascular density), because previous studies revelated that SRSF1 could regulate the AS of VEGF (vascular endothelial growth factor) and its different activities and its role in cancer progression $[8,10]$, we then correlated histological features with clinical data.

We found that SRSF1 and MVD were more expressed in patients with Pca than BPH and were also related to clinical features, in particular the expression of SRSF1, while high MVD was related both to worse OS and biochemical recurrence at 5 and 9 years in patients with Pca.

SRSF-1+ patients had worse 5- and 9-year biochemical recurrence (36\% and 6\%) with respect to SRSF-1- $(67 \%$ and $7 \% ; p<0.01)$ and similarly MVD+ patients $(44 \%$ and $7 \%)$ with respect to MVD- $(64 \%$ and $8 \% ; p<0.01)$. Restricting the analysis only to patients with PC and diabetes, we found that SRSF-1+ was associated with Ki-67+ (OR: 8.75; $p<0.05)$ and MVD+ (OR: 7.5; $p<0.05)$.

Regarding histological features, SRSF1 was related to overall MVD and with positive Ki67, the expression of Androgen Receptor (AR), Insulin Receptors (IR), insulin-like growth factor 1-receptor (IGF1-R), and the expression of prostate specific membrane antigen (PSMA); MVD was related to the expression of SRSF1 and also with positive Ki67, expression of Insulin Receptors (IR), (insulin-like growth factor 1-receptor) IGF1-R, and the expression of prostate specific membrane antigen (PSMA). In the sub-cohort of patients affected with PCa, SRSF1 was also related to the expression of AR, Ki67, IR and MVD [22]. In conclusion, several studies found a clinical and biological role of SRSF1 in PCa genesis, progression, and recurrence.

This evidence is summarized in Figure 2. 


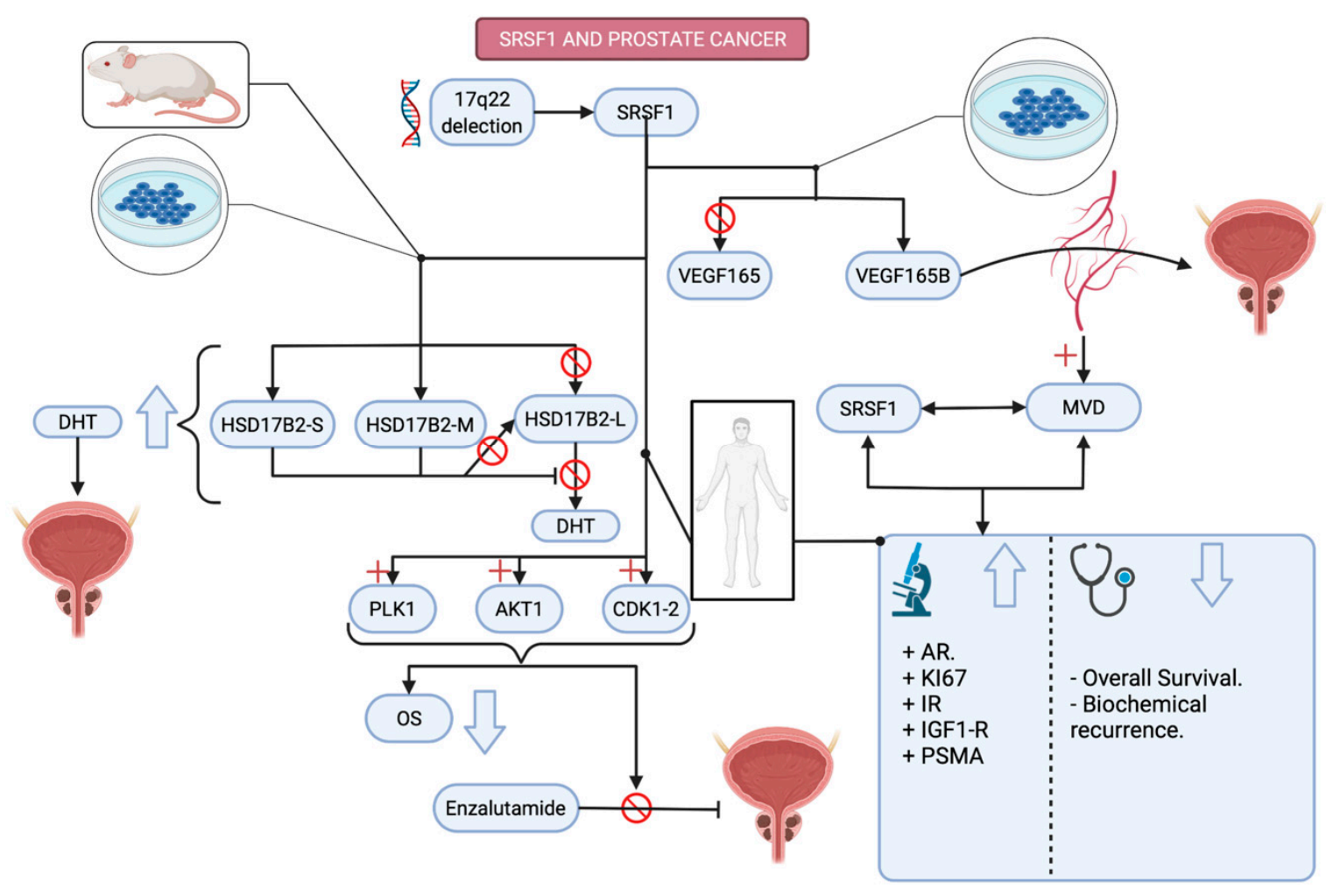

Figure 2. SRSF1 role and pathways in prostate cancer. Evidence from in vitro, animal, or clinical studies are specified with pictures. Created with Biorender.

\subsubsection{Breast Cancer}

Since 1999, some studies have postulated the role of SR proteins as alternative splicing factors involved in tumorigenesis and metastasis in breast cancer; in particular it was demonstrated that SR plays a key role in alternative splicing of CD44 [23], a gene with multiple exons and with significant variability of splicing; therefore the expression of CD44 variable exons 5, 6, 7 or 8 is related to metastasis in breast and ovarian cancers [24-26]. The role of SRSF1 in breast cancer has been investigated since 2015, when an in vitro study found a first cancer susceptibility candidate (CASC4), whose exon variable 9 is under the control of SRSF1, with alternative splicing events. Exon-9-included-CASC4 could have oncogenic effects because it encodes for a protein that increases acinar size and proliferation in breast tissue [3].

Another molecular study published in 2020 revealed that SRSF1 is not only an alternative splicing factor, but also has a crucial impact on IRES-mediated translation during the initiation of the mRNA in particular. In ER (-) breast cancer cells its role depends on growth conditions: under usual conditions, SRSF1 is located within the nucleus and it is involved in alternative splicing mechanisms and in the encoding of elements that also regulate the translation; however, under stress conditions, SRSF1 translocases from the nucleus to the cytoplasm where it plays a key role as an ITAF (Internal Ribosome Entry Site) and where it promotes MYC, and other mRNA, translation. On the other hand, on ER (+) breast cancer cells, SRSF1 plays a role depending on the cell cycle: during the G0 and G1 phases it modulates translation with alternative splicing mechanisms, during mitosis nucleus disassembling promotes the cytoplasmatic translocation of SRSF1 where it covers the role of an "ITAF", similar to that in ER (-) cells regulating the mitotic landscape [27].

SRSF1 also plays a direct role in promoting oncogenic pathways, in fact, in 2021 a study revealed that in breast cancer it is involved in alternative splicing promoting the overexpression of PTPMT1, which is related to breast cancer tumorigenesis through the P-AKT/C-MYC pathway [28]. 
A recent study published in 2021 revealed a direct clinical role of SRSF1 in breast cancer metastasis; in fact, this protein not only promotes the alternative splicing of oncogenic elements (C-MYC), but also could contribute to malignant progression and metastasis spread by stimulating the alternative splicing of DCUN1D5, including exon 4 that stabilizes the expression of this protein that is directly involved in metastatic breast cancer. The authors compared the expression level of DCUN1D5 in metastatic and non-metastatic breast cancer and also compared 5-year overall survival rates in patients with high and low DCUN1D5 expression levels; the authors found that DCUN1D5-exon-4, under the control of SRSF,1 was related to metastatic stage and worse prognosis in breast cancer [29].

This evidence is summarized in Figure 3.

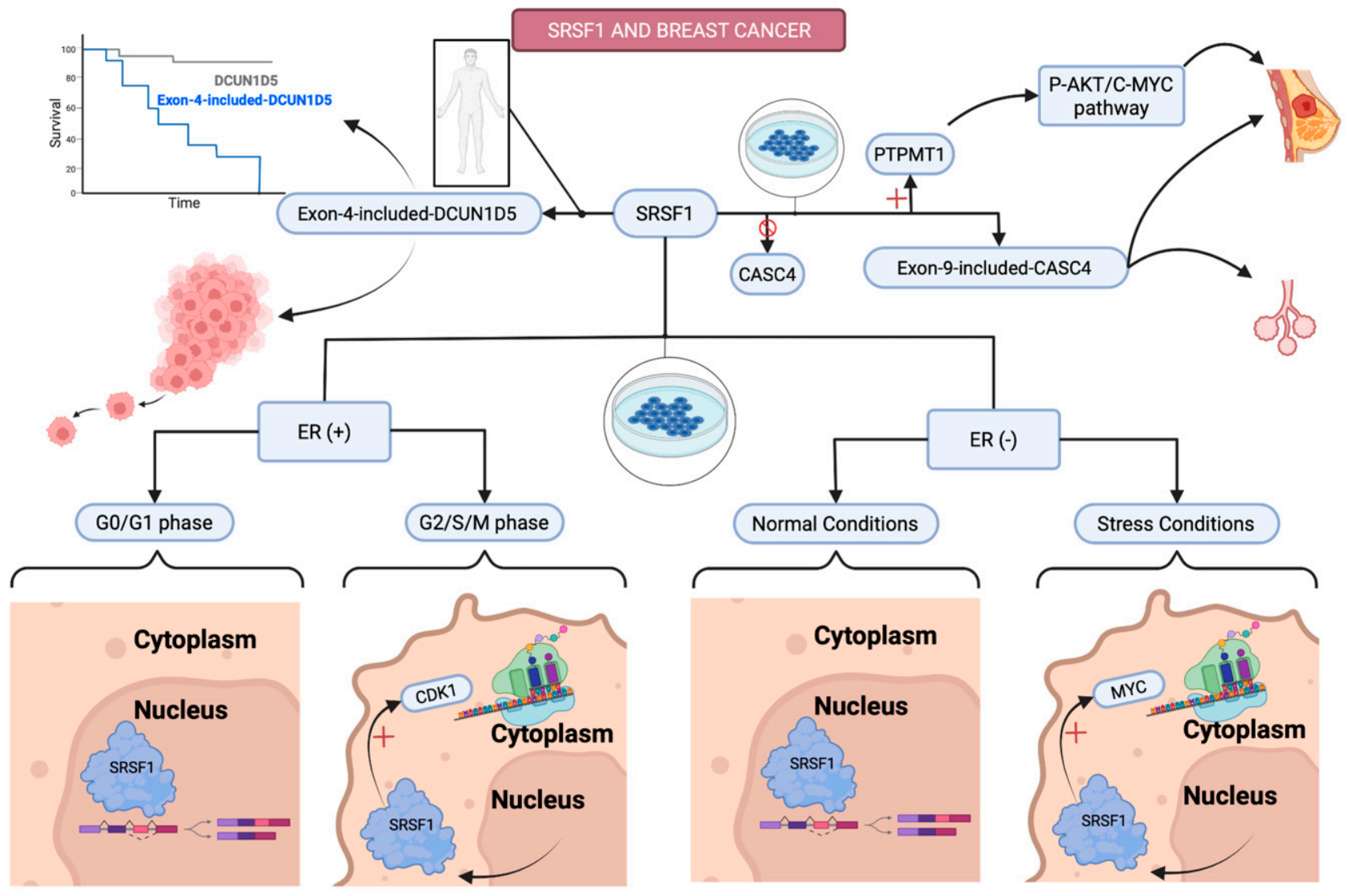

Figure 3. SRSF1 role and pathways in breast cancer. Evidence from in vitro, animal or clinical studies are specified with pictures. Created with Biorender. The Kaplan-Meier curves have only a figurative purpose.

\subsubsection{Lung Cancer}

Valid literature concerning the clinical role of SRSF1 in lung cancer started in 2010, when Ezponda T. et al. observed its key role in NSCLS (non-small cell lung cancer) through an mTORC1-dependent mechanism; the activation of the translational repressor 4E-BP1 and the activation of the surviving pathway seems to contribute to NSCLC progression [30]. Another in vitro study with cell culture of NSCLC and RNA transfection showed the role of Caspase 9 in chemotherapeutic sensitivity of non-small cell lung cancer; in particular, an alternative splicing event of Caspase 9, switching the expression ratio of Caspase 9a/9b from $9 a$ to $9 b$, caused an increasing of resistance from cisplatinum, daunorubicin and paclitaxel. This study also demonstrated that the mutation of the C9-I6/ISE enhancer element, leading to the overexpression of Caspase $9 \mathrm{~b}$, is mediated by the interaction of SRSF1 with C9-I6/ISE; therefore, the in vitro study postulated that SRSF1 could be a lung cancer chemoresistance mediator [31]. 
In 2012 Gout $S$ et al. published a paper on tissue samples from 53 lung SCCs (squamous cell carcinoma) and 54 ADCs (lung adenocarcinoma), 25 normal lung samples where included, the tissue samples underwent Immunohistochemistry analysis to explore the expression of SR Pre-mRNA splicing regulators and, in particular, of SRSF1. This study found that the overexpression of SRSF1 is associated with worse stage and diagnosis (III/IV) and with the presence of metastases (M+) only in NSCLC and, in particular, in ADC [32].

Jiang et al. [33] in 2016 published the first whole genomic and transcriptomic sequence from a Chinese population of 99 SCLCs (Small Cell Lung Carcinoma), correlating with clinical and survival data. This study was conducted by the application of Immunohistochemistry techniques; however, the finding of gain of copy number of SRSF1, and its correlation with poor survival rates in the population, led to a switch to a cell culture-study that confirmed that SRSF1 plays a key role in SCLC, too. They found that the overexpression of SRSF1 regulates the BCL2 pathway resulting in a lack of pro-apoptotic activity; furthermore, the knockdown of SRSF1 also caused an activation of caspase-3, similar to the cisplatin effect and the combination of SRSF1 knockdown and cisplatin caused a stronger caspase-3 activation than cisplatin alone. In this study it was found that SRSF1 can also play a key role in DNA-damaging signals in SCLC, in particular, suppressing PI3K/AKT and MEK/ERK pathways.

SRSF1 seems to be involved in lung cancer radio-sensitivity, too. In particular, a cell culture and in vivo animal study published in 2018 found that isoforms of PTPMT1 (short isoform and long isoform) could play a role in radio-resistance and sensitivity in lung cancer in mice. SRSF1 was proven to be able to regulate an alternative splicing of PTPMT1 promoting overexpression of long isoform of that protein that can reduce expression of MAPK and reduce the DNA-damage response to radiotherapy. This study also hypothesized SRSF1 in control of several other candidates involved in radio-sensitivity pathways [34].

In 2018 a paper published by Martinez-Terroba E. et al. demonstrated that SRSF1 regulates translation and/or stability of DNA ligase 1 (LIG1) by a mechanism depending on the activation of mTOR [35]. LIG1 is a DNA repair gene, in this study the authors demonstrated that LIG1 is a marker for poor prognosis in NSCLC, in particular, the authors found a correlation with a shorter Disease Free Survival (DFS) and Overall Survival; furthermore, in the same study LIG1 was identified as an independent prognostic factor for NSCLC [35].

A whole-transcriptome analysis was performed in 2019 from samples of lung cancer (LC) tissue and adjacent normal lung tissue to find AS events. The authors of this work found that SRSF1 plays a key role in the regulation of HIF (Hypoxia-inducible factors) alternative splicing, leading to a shift from HIF-1alfaL to HIF-1alfaS; in conclusion, the authors examined migration and invasion of cells in vitro with HIF-1alfa-targeting vectors and they found that isoform HIF-1alfaS has a greater metastatic potential in LC cells. They found that SRSF1 expression could play a clinical role enhancing metastatic activities of LC [36].

Another role of SRSF1 on NSCLC is to regulate an aberrant alternative splicing of the protein BIN1 (Bridging Integrator 1), a protein that could suppress immune escape in NSCLC and malignant behaviors [37]; in particular, SRSF1 activates the expression of the BIN1+12a isoform through 12a exon inclusion. The authors demonstrate that this aberrant isoform eliminates NSCLC cell apoptosis and suppression of invasion, leading to neoplasia free progression [38].

The most recent finding regarding SRSF1 and LC is the role of this proto-oncogene in autophagy, which is an intracellular degradation system that has the role of recycling and destroying old and aberrant proteins and organelles [39]. The authors demonstrated that SRSF1 regulates the alternative splicing of the long isoform of the protein Bcl-X, well known for play a role in cell survival [40]; the Bcl-X long isoform suppresses apoptosis interacting with the Beclin-1 protein and preventing Beclin1-PIK3C3 interaction. Beclin1-PIKeC3 interaction is a well-known complex that stimulates the formation of autophagosome 
and autophagy, playing a key clinical role in several settings such as tumorigenesis and neurodegeneration [41-43]. It was demonstrated that SRSF1 mediates the alternative splicing of the Bcl-X long isoform that suppresses apoptosis interacting with Beclin-1 protein and preventing Beclin1-PIK3C3 interaction, furthermore, SRSF1 interacts directly with PIK3C3 to disrupt this interaction [44]. In conclusion SRSF1 plays a key clinical role in tumorigenesis and progression for several subtypes of lung cancer with various mechanisms of action and interferes with different pathways.

This evidence is summarized in Figure 4.

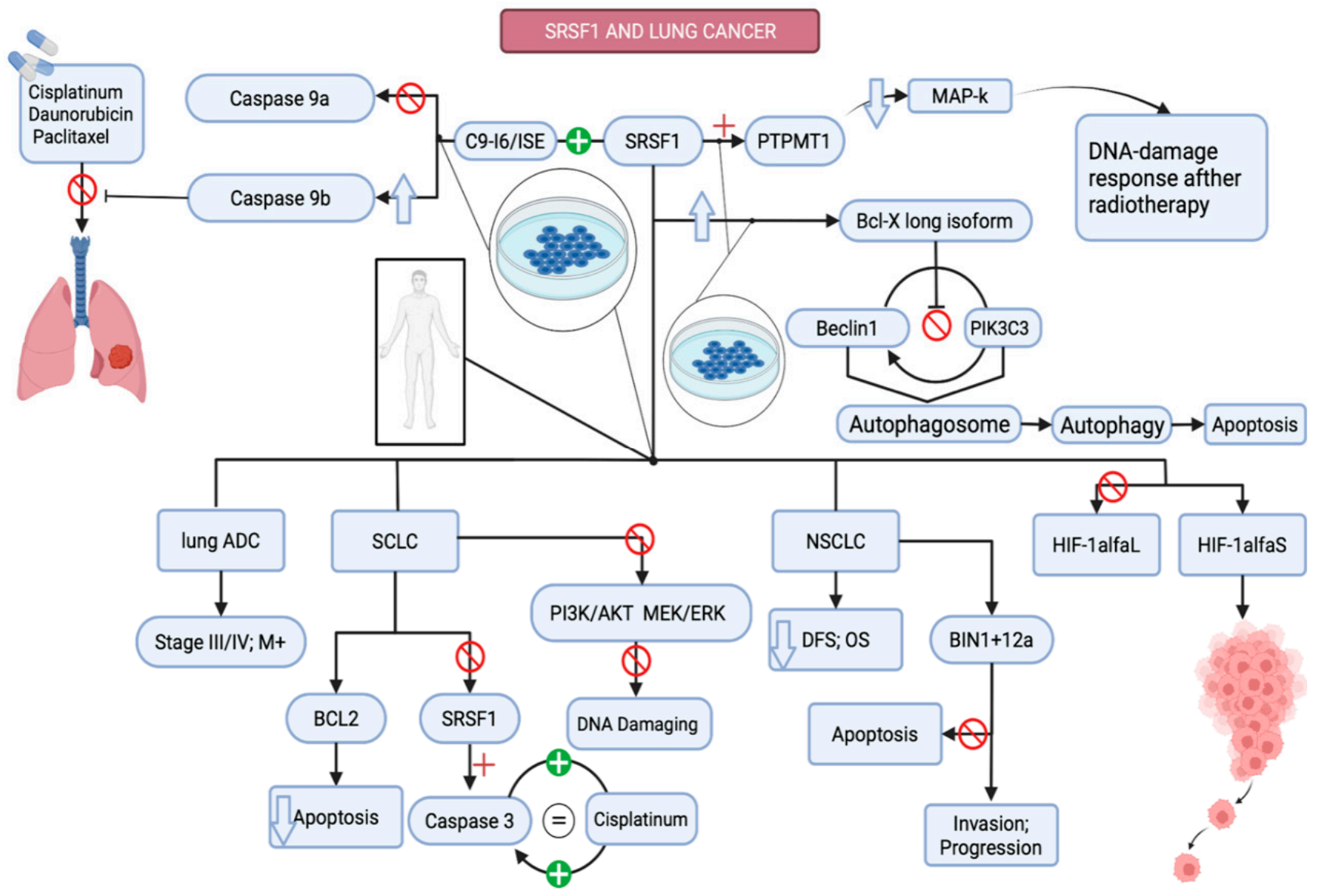

Figure 4. SRSF1 role and pathways in lung cancer. Evidence from in vitro, animal or clinical studies are specified with pictures. Created with Biorender.

\subsubsection{Colorectal Cancer}

A particularly important study was published in 2017 regarding the biological role of SRSF1 in colorectal cancer. A bioinformatics analysis was performed and the authors of this paper found that SRSF1 is related to several genes involved in colorectal cancer development and, in particular, they play a role in the cell cycle, such as in spliceosome, ubiquitin-mediated proteolysis, nucleotide excision repair, p53 pathway, DNA replication and RNA degradation. Further analysis found that SRSF1 is overexpressed in several types of colorectal cancer with a higher concentration in neoplastic tissue rather than in healthy tissue. A total of 468 SRSF1-related genes were found to be involved in colorectal cancers by overlapping 2678 SRSF1-related genes and 3625 colorectal cancer-related genes [45].

Another study, performed in vitro and in vivo with a xenograft model in mice and also a clinical study, were published in 2017 and found several relationships between SRSF1 and colon cancer [46]. They investigated the role of AS events for the DBF4B subunit (DBF4 zinc finger B) of the cyclin Cdc7 Kinase that regulates DNA replication during the cell cycle. The expression of DBF4B is regulated during the cell cycle and in particular its 
inhibition results in the attenuation of cell growth and the expression of markers of DNA damage; DBF4B mRNA levels are high during the $S$ phase of the cell cycle and low during the G1 phase [47]. The authors of this paper found that SRSF1 regulates, through AS, the inclusion of exon 6 in DBF4B (DBF4B-FL). To investigate the role of SRSF1 and DBF4B-FL in tumorigenesis in vivo, a xenograft model in mice with colon cancer cells (RKO) was performed; in particular, shRNAs targeted against DBF4B-FL and SRSF1 were introduced to knock them down in RKO cells and RKO/shDBF4B-FL and RKO/shSRSF1 were used to perform a xenograft. Both tests showed that DBF4B-FL and SRSF1 KD lead to a significant inhibition of cell growth.

To investigate the clinical role of DBF4B-FL in colon cancer, the authors compared the ratio of DBF4B FL/S mRNA in clinical CRC samples, examining sixty human CRC tissues paired with their noncancerous tissues. Furthermore, both SRSF1 and DBF4B-FL were highly expressed in neoplastic cells with respect to noncancerous tissue. The authors then investigated the expression of SRSF1 and its AS product in colon cancer cells. They found that the expression of SRSF1/DBF4B-FL was correlated to higher tumor grade and, furthermore, at the Kaplan-Meier analysis and Cox regression SRSF1 and DBF4B-FL were related to worse survival rates and where also both independent prognostic factors for colon cancer [46].

Comparable results with the previous work were found in 2021 in a study that confirmed SRSF1 as an independent prognostic factor in colorectal cancer, the same as the TNM classification. These results were also related to SRSF1 as an alternative splicing factor involved in the regulation of events such as MAPK interacting with Mnk2 (serine/threonine protein kinase 2), encoded by the MKNK2 gene. This protein plays a critical role in the MAPK pathway and can enhance tumorigenesis and proliferation [48].

We can affirm that SRSF1 also plays an important clinical role in the prognosis and tumor development for colon cancer.

This evidence is summarized in Figure 5.

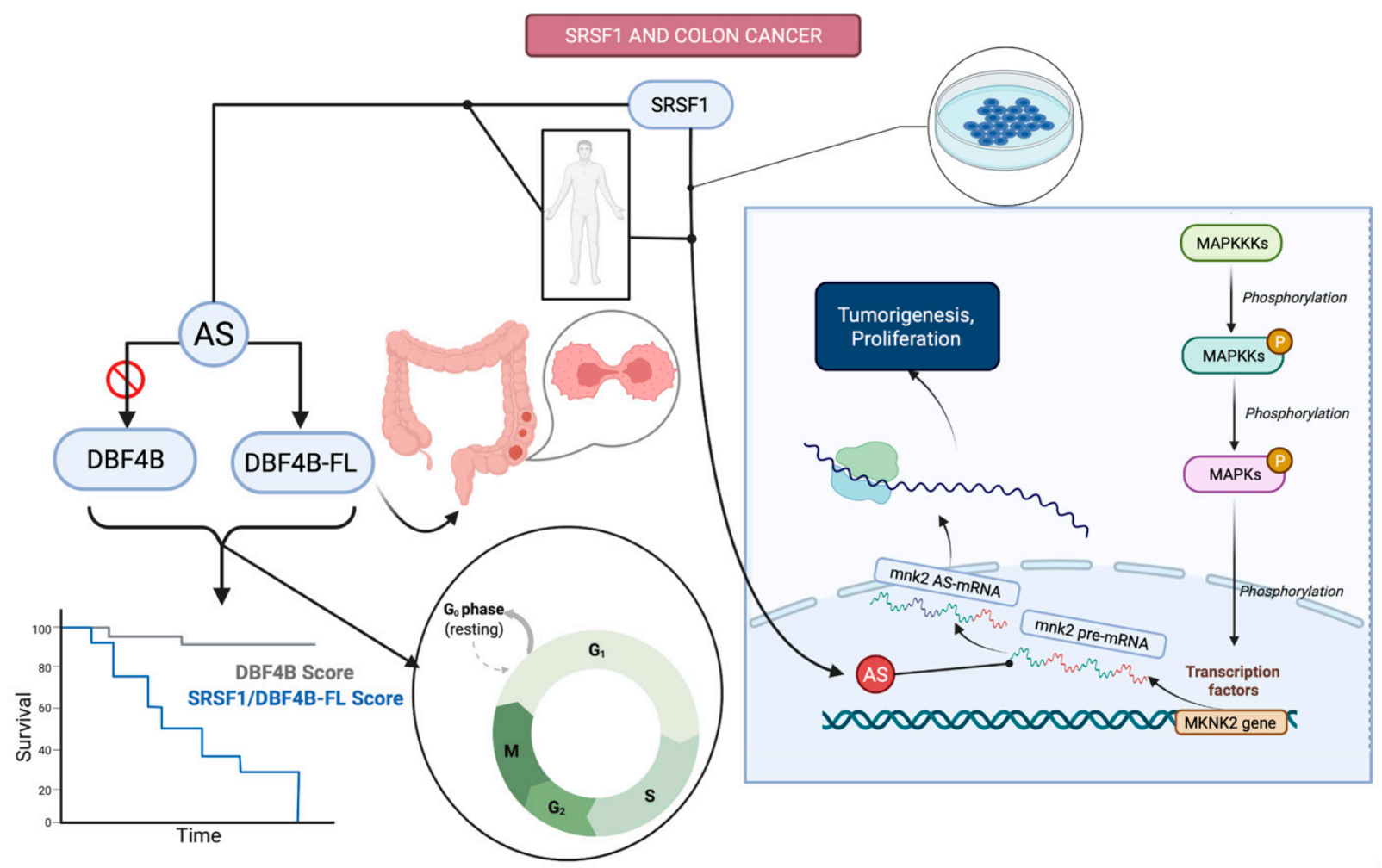

Figure 5. SRSF1 role and pathways in colorectal cancer. Evidence from in vitro, animal or clinical studies are specified with pictures. Created with Biorender. The Kaplan-Meier curves have only a figurative purpose. 


\subsubsection{Glioblastoma Multiforme}

Interesting papers have been published in the last few years regarding glioblastoma multiforme (GBM), an aggressive cancer of the brain. Consequently, with the growing interest in circRNAs (circular RNAs), a new class of RNAs that are highly expressed in the brain, studies have investigated the role of hsa_circ_0001445 (circSMARCA5); in particular, it was found that circSMARCA5 interacted with SRSF1 splicing factor, modulating its activity in GBM cells. Studies have been conducted on sampling biopsies from GBM and surrounding healthy brain tissues collected in a single institution; thereafter, histological and molecular features were correlated with clinical data. In particular, it was found that circSMARCA5 expression was downregulated in GBM, compared to healthy tissue, and the decreased expression of circSMARCA5 matched with a crescent grade of neoplasia malignancy and an in vitro reduce migration-capability of GBM cells [49]. Moreover, the role of circSMARCA5 as a splicing regulator in GBM was investigated and it was revealed that it interacts with SRSF1 leading to an inhibition of this AS factor; in fact, it was found that SRSF1 is upregulated when circSMARCA5 is downregulated in association with an upregulation of VEGF, suggesting that SRSF1 could upregulate the expression of VEGF leading to a more angiogenic activity, cancer progression and sprouting. In this study clinical data suggested that MVD, VEGF and SRSF1 correlate with poorer OS, and progression-free survival (PFS) [50,51]. In conclusion, there is a key clinical role of SRSF1, in association with its regulator circSMARCA5, in cell migration and angiogenic potential for GBM. The interaction between SRSF1 and circSMARCA5 is a key factor to try to individuate new possible pharmacological or gene therapies for GBM, with this perspective it was found that the "GAUGAA" motif in circSMARCA5 is responsible for the binding of SRSF1 and enhancing the angiogenic potential and cell migration of GBM [52].

This evidence is summarized in Figure 6.

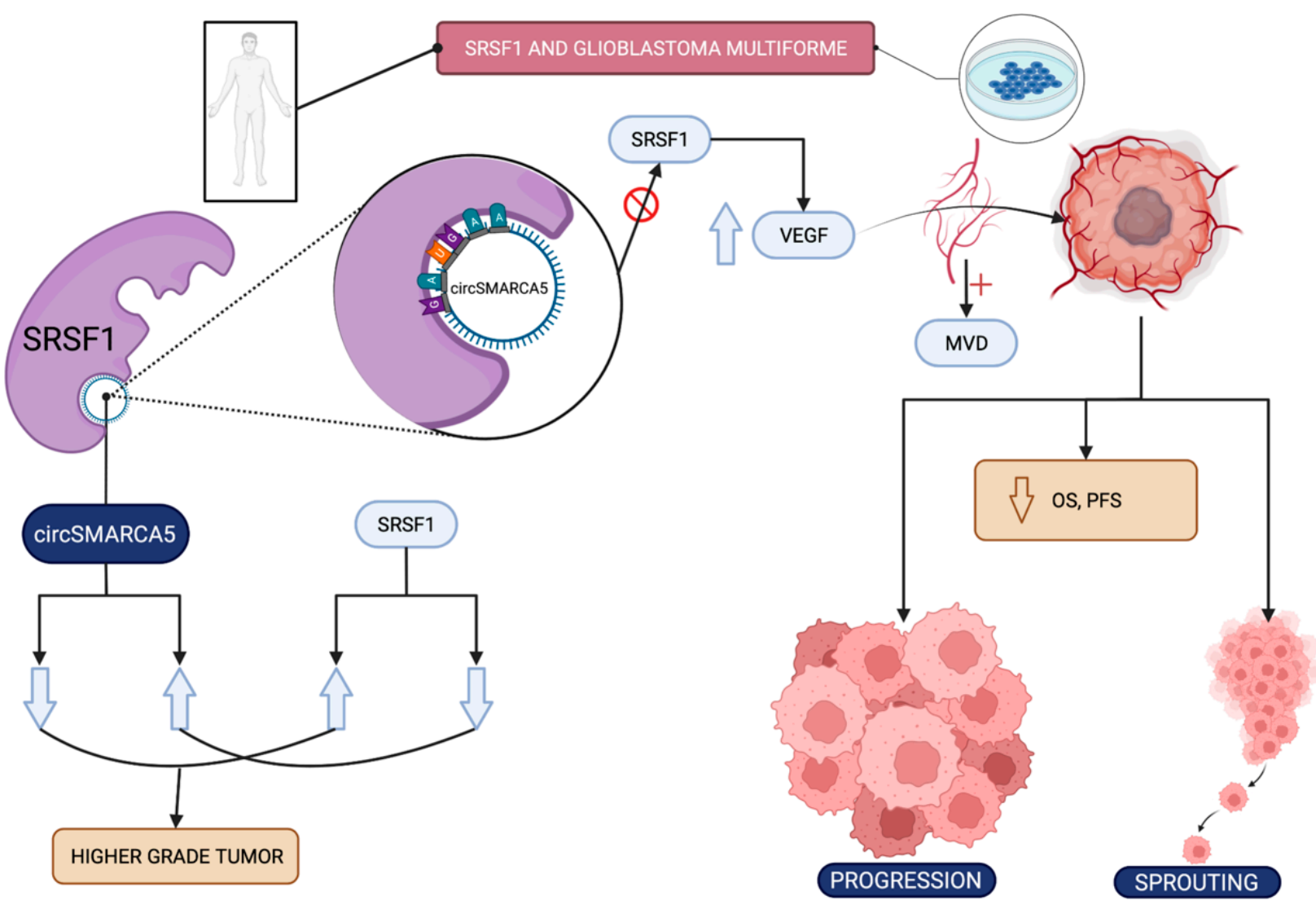

Figure 6. SRSF1 role and pathways in glioblastoma multiforme. Evidence from in vitro, animal or clinical studies are specified with pictures. Created with Biorender. 


\section{Conclusions}

According to our review, SRSF1 has been demonstrated to be overexpressed in a wide range of cancer tissues and to take part in tumorigenesis and progression through different mechanisms. Although more studies should be conducted in this field, SRSF1 may be an interesting biomarker able to predict oncological outcomes in many tumors, in particular in prostate cancer and Glioblastoma Multiforme. Thus, the correct identification in pathological tissue could help clinicians to personalize follow-up and therapies.

We think that the literature evidence in this review is only the tip of the iceberg and, certainly, alternative splicing and SRSF1 could play a role in every cancer. High expression of SRSF1 is associated with a worse prognosis in PCa and copy loss of 17q22 is associated with lower survival.

In breast cancer, SRSF1 moves to the cytoplasm where it promotes the translation of MYC and other mRNAs; moreover, in ER (+) breast cancer, SRSF1 seems to be implicated in cell cycle regulation, while in lung cancer SRSF1 may act in determining chemoresistance.

We hope that this review can be the starting point to study better the role of SRSF1 and relative pathway, to find new information concerning cancer biology and therapies.

Author Contributions: Conceptualization, A.L.G., G.B. and G.I.R.; methodology, A.L.G.; formal analysis, A.L.G.; investigation, A.L.G. and M.G.A.; resources, A.L.G. and M.G.A.; data curation, A.L.G.; writing—original draft preparation, A.L.G. and M.G.A.; writing-review and editing, A.L.G. and G.I.R.; visualization, S.C.; supervision, G.I.R., E.D.T., G.M. (Giuseppe Morgia), O.d.C., M.F., S.L., G.M. (Gennaro Musi) and S.C. All authors have read and agreed to the published version of the manuscript.

Funding: This research received no external funding.

Institutional Review Board Statement: Not applicable.

Informed Consent Statement: Not applicable.

Data Availability Statement: Not applicable.

Conflicts of Interest: The authors declare no conflict of interest.

\section{References}

1. Long, J.C.; Caceres, J.F. The SR protein family of splicing factors: Master regulators of gene expression. Biochem. J. 2009, 417, 15-27. [CrossRef] [PubMed]

2. Howard, J.M.; Sanford, J.R. The RNAissance family: SR proteins as multifaceted regulators of gene expression. Wiley Interdiscip. Rev. RNA 2015, 6, 93-110. [CrossRef] [PubMed]

3. Anczuków, O.; Akerman, M.; Cléry, A.; Wu, J.; Shen, C.; Shirole, N.H.; Raimer, A.; Sun, S.; Jensen, M.A.; Hua, Y.; et al. SRSF1-Regulated Alternative Splicing in Breast Cancer. Mol. Cell 2015, 60, 105-117. [CrossRef] [PubMed]

4. Ngo, J.C.K.; Chakrabarti, S.; Ding, J.-H.; Velazquez-Dones, A.; Nolen, B.; Aubol, B.E.; Adams, J.A.; Fu, X.-D.; Ghosh, G. Interplay between SRPK and Clk/Sty Kinases in Phosphorylation of the Splicing Factor ASF/SF2 Is Regulated by a Docking Motif in ASF/SF2. Mol. Cell 2005, 20, 77-89. [CrossRef]

5. Huang, Y.; Yario, T.A.; Steitz, J.A. A molecular link between SR protein dephosphorylation and mRNA export. Proc. Natl. Acad. Sci. USA 2004, 101, 9666-9670. [CrossRef]

6. Srebrow, A.; Kornblihtt, A.R. The connection between splicing and cancer. J. Cell Sci. 2006, 119, 2635-2641. [CrossRef] [PubMed]

7. Das, S.; Krainer, A.R. Emerging Functions of SRSF1, Splicing Factor and Oncoprotein, in RNA Metabolism and Cancer. Mol. Cancer Res. 2014, 12, 1195-1204. [CrossRef]

8. Karni, R.; de Stanchina, E.; Lowe, S.W.; Sinha, R.; Mu, D.; Krainer, A.R. The gene encoding the splicing factor SF2/ASF is a proto-oncogene. Nat. Struct. Mol. Biol. 2007, 14, 185-193. [CrossRef]

9. Zhou, X.; Wang, R.; Li, X.; Yu, L.; Hua, D.; Sun, C.; Shi, C.; Luo, W.; Rao, C.; Jiang, Z.; et al. Splicing factor SRSF1 promotes gliomagenesis via oncogenic splice-switching of MYO1B. J. Clin. Investig. 2019, 129, 676-693. [CrossRef]

10. Anczuków, O.; Rosenberg, A.Z.; Akerman, M.; Das, S.; Zhan, L.; Karni, R.; Muthuswamy, S.K.; Krainer, A.R. The splicing factor SRSF1 regulates apoptosis and proliferation to promote mammary epithelial cell transformation. Nat. Struct. Mol. Biol. 2012, 19, 220-228. [CrossRef]

11. Das, S.; Anczuków, O.; Akerman, M.; Krainer, A.R. Oncogenic Splicing Factor SRSF1 Is a Critical Transcriptional Target of MYC. Cell Rep. 2012, 1, 110-117. [CrossRef] [PubMed] 
12. Arzalluz-Luque, Á.; Cabrera, J.L.; Skottman, H.; Benguria, A.; Bolinches-Amorós, A.; Cuenca, N.; Lupo, V.; Dopazo, A.; Tarazona, S.; Delás, B.; et al. Mutant PRPF8 Causes Widespread Splicing Changes in Spliceosome Components in Retinitis Pigmentosa Patient iPSC-Derived RPE Cells. Front. Neurosci. 2021, 15, 8. [CrossRef]

13. Cook, D.A.; Reed, D.A. Appraising the Quality of Medical Education Research Methods. Acad. Med. 2015, 90, 1067-1076. [CrossRef] [PubMed]

14. Wells, G.; Shea, B.; O'Connell, D.; Robertson, J.; Peterson, J.; Welch, V.; Losos, M.; Tugwell, P. The Newcastle-Ottawa scale (NOS) for assessing the quality of nonrandomised studies in meta-analyses. Ott. Ott. Hosp. Res. Inst. 2011, 1-12. Available online: www.ohri.ca/programs/clinical_epidemiology/oxford.asp (accessed on 18 December 2021).

15. Ferrer, F.A.; Miller, L.J.; Andrawis, R.I.; Kurtzman, S.H.; Albertsen, P.C.; Laudone, V.P.; Kreutzer, D.L. Angiogenesis and prostate cancer: In vivo and in vitro expression of angiogenesis factors by prostate cancer cells. Urology 1998, 51, 161-167. [CrossRef]

16. Ferrer, F.A.; Miller, L.J.; Andrawis, R.I.; Kurtzman, S.H.; Albertsen, P.C.; Laudone, V.P.; Kreutzer, D.L. Vascular endothelial growth factor (VEGF) expression in human prostate cancer: In situ and in vitro expression of VEGF by human prostate cancer cells. J. Urol. 1997, 157, 2329-2333. [CrossRef]

17. Mavrou, A.; Brakspear, K.; Hamdollah-Zadeh, M.; Damodaran, G.; Babaei-Jadidi, R.; Oxley, J.; Gillatt, D.A.; Ladomery, M.R.; Harper, S.J.; Bates, D.O.; et al. Serine-arginine protein kinase 1 (SRPK1) inhibition as a potential novel targeted therapeutic strategy in prostate cancer. Oncogene 2015, 34, 4311-4319. [CrossRef]

18. Oltean, S.; Gammons, M.; Hulse, R.; Hamdollah-Zadeh, M.; Mavrou, A.; Donaldson, L.; Salmon, A.H.; Harper, S.J.; Ladomery, M.R.; Bates, D.O. SRPK1 inhibition in vivo: Modulation of VEGF splicing and potential treatment for multiple diseases. Biochem. Soc. Trans. 2012, 40, 831-835. [CrossRef]

19. Amin, E.M.; Oltean, S.; Hua, J.; Gammons, M.V.R.; Hamdollah-Zadeh, M.; Welsh, G.I.; Cheung, M.-K.; Ni, L.; Kase, S.; Rennel, E.S.; et al. WT1 mutants reveal SRPK1 to be a downstream angiogenesis target by altering VEGF splicing. Cancer Cell 2011, 20, 768-780. [CrossRef]

20. Gao, X.; Dai, C.; Huang, S.; Tang, J.; Chen, G.; Li, J.; Zhu, Z.; Zhu, X.; Zhou, S.; Gao, Y.; et al. Functional Silencing of HSD17B2 in Prostate Cancer Promotes Disease Progression. Clin. Cancer Res. 2019, 25, 1291-1301. [CrossRef]

21. Guan, X.; Sun, D.; Lu, E.; Urrutia, J.A.; Reiter, R.E.; Rettig, M.; Evans, C.P.; Lara, P.; Gleave, M.; Beer, T.M.; et al. Copy Number Loss of 17q22 Is Associated with Enzalutamide Resistance and Poor Prognosis in Metastatic Castration-Resistant Prostate Cancer. Clin. Cancer Res. 2020, 26, 4616-4624. [CrossRef] [PubMed]

22. Broggi, G.; Lo Giudice, A.; Di Mauro, M.; Asmundo, M.G.; Pricoco, E.; Piombino, E.; Caltabiano, R.; Morgia, G.; Russo, G.I. SRSF-1 and microvessel density immunohistochemical analysis by semi-automated tissue microarray in prostate cancer patients with diabetes (DIAMOND study). Prostate 2021, 81, 882-892. [CrossRef] [PubMed]

23. Stickeler, E.; Kittrell, F.; Medina, D.; Berget, S.M. Stage-specific changes in SR splicing factors and alternative splicing in mammary tumorigenesis. Oncogene 1999, 18, 3574-3582. [CrossRef] [PubMed]

24. Stickeler, E.; Runnebaum, I.B.; Möbus, V.J.; Kieback, D.G.; Kreienberg, R. Expression of CD44 standard and variant isoforms v5, v6 and v7 in human ovarian cancer cell lines. Anticancer Res. 1997, 17, 1871-1876. [PubMed]

25. Heider, K.-H.; Mulder, J.-W.R.; Ostermann, E.; Susani, S.; Patzelt, E.; Pals, S.T.; Adolf, G.R. Splice variants of the cell surface glycoprotein CD44 associated with metastatic tumour cells are expressed in normal tissues of humans and cynomolgus monkeys. Eur. J. Cancer 1995, 31, 2385-2391. [CrossRef]

26. Sinn, H.-P.; Heider, K.-H.; Skroch-Angel, P.; von Minckwitz, G.; Kaufmann, M.; Herrlich, P.; Ponta, H. Human mammary carcinomas express homologues of rat metastasis-associated variants of CD44. Breast Cancer Res. Treat. 1995, 36, 307-313. [CrossRef]

27. Vaklavas, C.; Blume, S.W.; Grizzle, W.E. Hallmarks and Determinants of Oncogenic Translation Revealed by Ribosome Profiling in Models of Breast Cancer. Transl. Oncol. 2020, 13, 452-470. [CrossRef]

28. Du, J.X.; Luo, Y.H.; Zhang, S.J.; Wang, B.; Chen, C.; Zhu, G.Q.; Zhu, P.; Cai, C.Z.; Wan, J.L.; Cai, J.L.; et al. Splicing factor SRSF1 promotes breast cancer progression via oncogenic splice switching of PTPMT1. J. Exp. Clin. Cancer Res. 2021, 40, 171. [CrossRef]

29. Oh, J.; Pradella, D.; Shao, C.; Li, H.; Choi, N.; Ha, J.; Ruggiero, S.; Fu, X.D.; Zheng, X.; Ghigna, C.; et al. Widespread alternative splicing changes in metastatic breast cancer cells. Cells 2021, 10, 858. [CrossRef]

30. Ezponda, T.; Pajares, M.J.; Agorreta, J.; Echeveste, J.I.; López-Picazo, J.M.; Torre, W.; Pio, R.; Montuenga, L.M. The oncoprotein SF2/ASF promotes non-small cell lung cancer survival by enhancing survivin expression. Clin. Cancer Res. 2010, 16, 4113-4125. [CrossRef]

31. Shultz, J.C.; Goehe, R.W.; Murudkar, C.S.; Wijesinghe, D.S.; Mayton, E.K.; Massiello, A.; Hawkins, A.J.; Mukerjee, P.; Pinkerman, R.L.; Park, M.A.; et al. SRSF1 Regulates the Alternative Splicing of Caspase 9 Via A Novel Intronic Splicing Enhancer Affecting the Chemotherapeutic Sensitivity of Non-Small Cell Lung Cancer Cells. Mol. Cancer Res. 2011, 9, 889-900. [CrossRef] [PubMed]

32. Gout, S.; Brambilla, E.; Boudria, A.; Drissi, R.; Lantuejoul, S.; Gazzeri, S.; Eymin, B. Abnormal Expression of the Pre-mRNA Splicing Regulators SRSF1, SRSF2, SRPK1 and SRPK2 in Non Small Cell Lung Carcinoma. PLoS ONE 2012, 7, e46539. [CrossRef] [PubMed]

33. Jiang, L.; Huang, J.; Higgs, B.W.; Hu, Z.; Xiao, Z.; Yao, X.; Conley, S.; Zhong, H.; Liu, Z.; Brohawn, P.; et al. Genomic Landscape Survey Identifies SRSF1 as a Key Oncodriver in Small Cell Lung Cancer. PLoS Genet. 2016, 12, e1005895. [CrossRef]

34. Sheng, J.; Zhao, Q.; Zhao, J.; Zhang, W.; Sun, Y.; Qin, P.; Lv, Y.; Bai, L.; Yang, Q.; Chen, L.; et al. SRSF1 modulates PTPMT1 alternative splicing to regulate lung cancer cell radioresistance. EBioMedicine 2018, 38, 113-126. [CrossRef] [PubMed] 
35. Martínez-Terroba, E.; Ezponda, T.; Bértolo, C.; Sainz, C.; Remírez, A.; Agorreta, J.; Garmendia, I.; Behrens, C.; Pio, R.; Wistuba, I.I.; et al. The oncogenic RNA-binding protein SRSF1 regulates LIG1 in non-small cell lung cancer. Lab. Investig. 2018, 98, 1562-1574. [CrossRef] [PubMed]

36. Chang, H.L.; Lin, J.C. SRSF1 and RBM4 differentially modulate the oncogenic effect of HIF- $1 \alpha$ in lung cancer cells through alternative splicing mechanism. Biochim. Biophys. Acta-Mol. Cell Res. 2019, 1866, 118550. [CrossRef]

37. Wang, J.; Jia, Y.; Zhao, S.; Zhang, X.; Wang, X.; Han, X.; Wang, Y.; Ma, M.; Shi, J.; Liu, L. BIN1 reverses PD-L1-mediated immune escape by inactivating the c-MYC and EGFR/MAPK signaling pathways in non-small cell lung cancer. Oncogene 2017, 36, 6235-6243. [CrossRef]

38. Wang, J.; Liu, T.; Wang, M.; Lv, W.; Wang, Y.; Jia, Y.; Zhang, R.; Liu, L. SRSF1-dependent alternative splicing attenuates BIN1 expression in non-small cell lung cancer. J. Cell. Biochem. 2020, 121, 946-953. [CrossRef]

39. Yorimitsu, T.; Klionsky, D.J. Autophagy: Molecular machinery for self-eating. Cell Death Differ. 2005, 12, 1542-1552. [CrossRef]

40. Boise, L.H.; González-García, M.; Postema, C.E.; Ding, L.; Lindsten, T.; Turka, L.A.; Mao, X.; Nuñez, G.; Thompson, C.B. bcl-x, a bcl-2-related gene that functions as a dominant regulator of apoptotic cell death. Cell 1993, 74, 597-608. [CrossRef]

41. Peng, Y.; Miao, H.; Wu, S.; Yang, W.; Zhang, Y.; Xie, G.; Xie, X.; Li, J.; Shi, C.; Ye, L.; et al. ABHD5 interacts with BECN1 to regulate autophagy and tumorigenesis of colon cancer independent of PNPLA2. Autophagy 2016, 12, 2167-2182. [CrossRef] [PubMed]

42. Morselli, E.; Galluzzi, L.; Kepp, O.; Vicencio, J.-M.; Criollo, A.; Maiuri, M.C.; Kroemer, G. Anti- and pro-tumor functions of autophagy. Biochim. Biophys. Acta-Mol. Cell Res. 2009, 1793, 1524-1532. [CrossRef] [PubMed]

43. Maria Fimia, G.; Stoykova, A.; Romagnoli, A.; Giunta, L.; Di Bartolomeo, S.; Nardacci, R.; Corazzari, M.; Fuoco, C.; Ucar, A.; Schwartz, P.; et al. Ambra1 regulates autophagy and development of the nervous system. Nature 2007, 447, 1121-1125. [CrossRef] [PubMed]

44. Lv, Y.; Zhang, W.; Zhao, J.; Sun, B.; Qi, Y.; Ji, H.; Chen, C.; Zhang, J.; Sheng, J.; Wang, T.; et al. SRSF1 inhibits autophagy through regulating Bcl-x splicing and interacting with PIK3C3 in lung cancer. Signal. Transduct. Target. Ther. 2021, 6, 108. [CrossRef] [PubMed]

45. Sheng, J.; Zhao, J.; Xu, Q.; Wang, L.; Zhang, W.; Zhang, Y. Bioinformatics analysis of SRSF1-controlled gene networks in colorectal cancer. Oncol. Lett. 2017, 14, 5393-5399. [CrossRef]

46. Chen, L.; Luo, C.; Shen, L.; Liu, Y.; Wang, Q.; Zhang, C.; Guo, R.; Zhang, Y.; Xie, Z.; Wei, N.; et al. SRSF1 Prevents DNA Damage and Promotes Tumorigenesis through Regulation of DBF4B Pre-mRNA Splicing. Cell Rep. 2017, 21, 3406-3413. [CrossRef]

47. Montagnoli, A.; Bosotti, R.; Villa, F.; Rialland, M.; Brotherton, D.; Mercurio, C.; Berthelsen, J.; Santocanale, C. Drf1, a novel regulatory subunit for human Cdc7 kinase. EMBO J. 2002, 21, 3171-3181. [CrossRef]

48. Liu, H.; Gong, Z.; Li, K.; Zhang, Q.; Xu, Z.; Xu, Y. SRPK1/2 and PP1 $\alpha$ exert opposite functions by modulating SRSF1-guided MKNK2 alternative splicing in colon adenocarcinoma. J. Exp. Clin. Cancer Res. 2021, 40, 75. [CrossRef]

49. Stella, M.; Falzone, L.; Caponnetto, A.; Gattuso, G.; Barbagallo, C.; Battaglia, R.; Mirabella, F.; Broggi, G.; Altieri, R.; Certo, F.; et al. Serum Extracellular Vesicle-Derived circHIPK3 and circSMARCA5 Are Two Novel Diagnostic Biomarkers for Glioblastoma Multiforme. Pharmaceuticals 2021, 14, 618. [CrossRef]

50. Barbagallo, D.; Caponnetto, A.; Brex, D.; Mirabella, F.; Barbagallo, C.; Lauretta, G.; Morrone, A.; Certo, F.; Broggi, G.; Caltabiano, R.; et al . CircSMARCA5 regulates VEGFA mRNA splicing and angiogenesis in glioblastoma multiforme through the binding of SRSF1. Cancers 2019, 11, 194. [CrossRef]

51. Broggi, G.; Salvatorelli, L.; Barbagallo, D.; Certo, F.; Altieri, R.; Tirrò, E.; Massimino, M.; Vigneri, P.; Guadagno, E.; Maugeri, G.; et al. Diagnostic Utility of the Immunohistochemical Expression of Serine and Arginine Rich Splicing Factor 1 (SRSF1) in the Differential Diagnosis of Adult Gliomas. Cancers 2021, 13, 2086. [CrossRef] [PubMed]

52. Barbagallo, D.; Caponnetto, A.; Barbagallo, C.; Battaglia, R.; Mirabella, F.; Brex, D.; Stella, M.; Broggi, G.; Altieri, R.; Certo, F.; et al. The gaugaa motif is responsible for the binding between circsmarca5 and srsf1 and related downstream effects on glioblastoma multiforme cell migration and angiogenic potential. Int. J. Mol. Sci. 2021, 22, 1678. [CrossRef] [PubMed] 